\title{
Validation of a CFD model of a single stage centrifugal compressor by mass-averaged parameters
}

\author{
Sergey Bogdanets, Vitaly Blinov, Viacheslav Sedunin, Oleg Komarov and Alexander
}

Skorohodov

UrFU, Department of Turbines and Engines, Yekaterinburg

\begin{abstract}
In this paper a validation of a computational model is presented for single-stage centrifugal compressor with an axial-flow impeller and a vaneless diffuser. The comparison was made by mass averaged performance. The paper shows that the model used is able to predict overall performance near the design operating point within $1.2 \%$ difference. However, further away the model fails to follow the experimental characteristics.
\end{abstract}

\section{Introduction}

Three-dimensional physical effects inside the rotor flow path of a centrifugal compressor must be accounted for when the high efficiency of the compressor is aimed. At present, the application of the methods of computational fluid dynamic (CFD) has become an integral part of various researches, and requirements to accuracy of such results are also increasing. This work is devoted to the validation of a computational model of a centrifugal compressor with an axial inlet impeller and a vaneless diffuser.

In [1] an overview of aerodynamic studies in centrifugal compressors is presented. Many studies support the evidence of flow separation at the suction side of the rotor even at design operating point. Therefore the so-called "jet-wake" model is widely applied. This model in most cases reliably describes the physics of the flow in a centrifugal compressor.

Many papers are devoted to the validation of numerical simulations [2, 3]. But modeling and validation of the flow in a centrifugal impeller with an axial inlet has not been researched as good as for simple axial and centrifugal compressors with radial blades.

In this work a flow in a single-stage centrifugal compressor is modeled by means of CFD code ANSYS CFX which is based on solving Reynolds-averaged Navier-Stokes equations (RANS).

One of the main difficulties with RANS methods is that they may fail to correctly predict the separation of the boundary layer. This leads to incorrect definition of the sizes of "jet" and "wake" zones, and, therefore, to a change in core flow parameters and massaveraged stage performance.

\section{Geometry description}


The experimental results, obtained by D. Eckardt et.al. in [4] were used for validation. An in-house code was used to create a 3D model of the compressor flow path (Figure 1a), geometry data from the report, partially shown on Figure $1 \mathrm{~b}$. The blade was made based on the law of blade angle distribution. The inlet external blade angle $\beta_{l e}$ is $27^{\circ}$, the outlet blade angle $\beta_{2}=90^{\circ}$. The radial tip varies from inlet to outlet, from $0.5 \mathrm{~mm}$ to $0.7 \mathrm{~mm}$, respectively. Blade thickness is constant in flow direction and equals to $3.5 \mathrm{~mm}$ and $3 \mathrm{~mm}$, for hub and shroud respectively. Leading and trailing edges were made circular with a radius of half of the blade thickness.
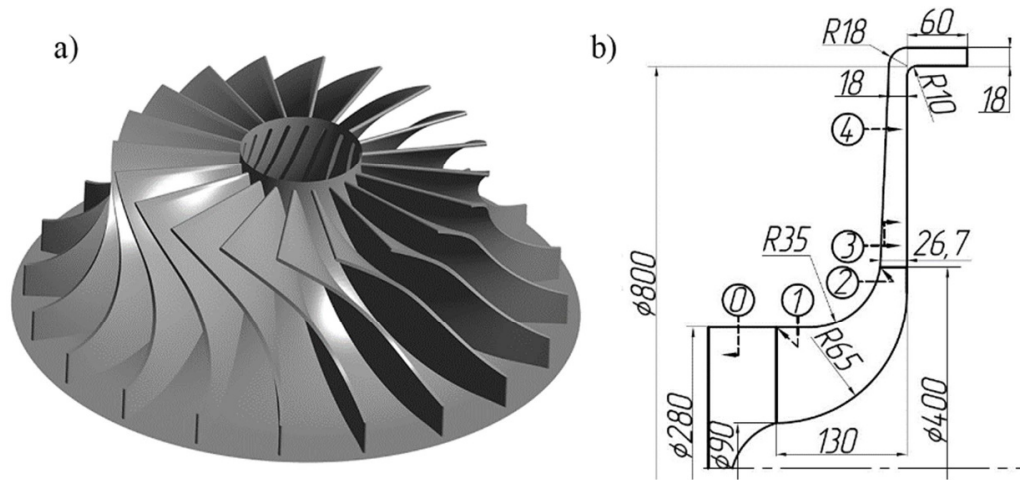

Fig. 1. a) Three-dimensional model of centrifugal compressor. b) Main dimensions and measuring points positions taken from [4], measurements in mm, 0-compressor intake, 1- impeller inlet edge, 2impeller outlet edge, 3- diffuser inlet region, 4- diffuser outlet.

\section{Computational model}

The boundary conditions were as: a total pressure of $101325 \mathrm{~Pa}$ and total temperature of $288 \mathrm{~K}$ at the inlet and mass flow at the diffuser outlet. These boundary conditions were chosen in order to have stable convergence above the design operating point. The radial tip with relative magnitude of about $0.5 \%$ to $2.6 \%$ was simulated by the GGI interface. Periodic boundary conditions were applied at the side faces of the model. To study the mixing of the jet-wake in the diffuser, a frozen rotor interface was set between rotating and stationary domains. The turbulence model $k-\varepsilon$ allows to reduce computational cost due to the coarser grid. Fluid - air ideal gas. Under conditions of limited computing power, the mesh size for both domains is taken in accordance with the parameter $y+$ requirement and is 540,000. Convergence criteria: below than $10^{-6}$ and stabilized value of the compressor efficiency.

\section{Results analysis}

Figure 2a shows the speed line of the compressor. It can be seen that numerical modelling predicts a lower compressor head in the entire operating range and an earlier onset of unstable operation. All computed speed lines have a pressure ratio error in the range 1-2\% as long as convergence criteria are satisfied. The lowest speed lines show better agreement. Mach number based on tip speed at design rotational speed is equal 1.11. Computed efficiency in the stably converging range is remarkably higher. 

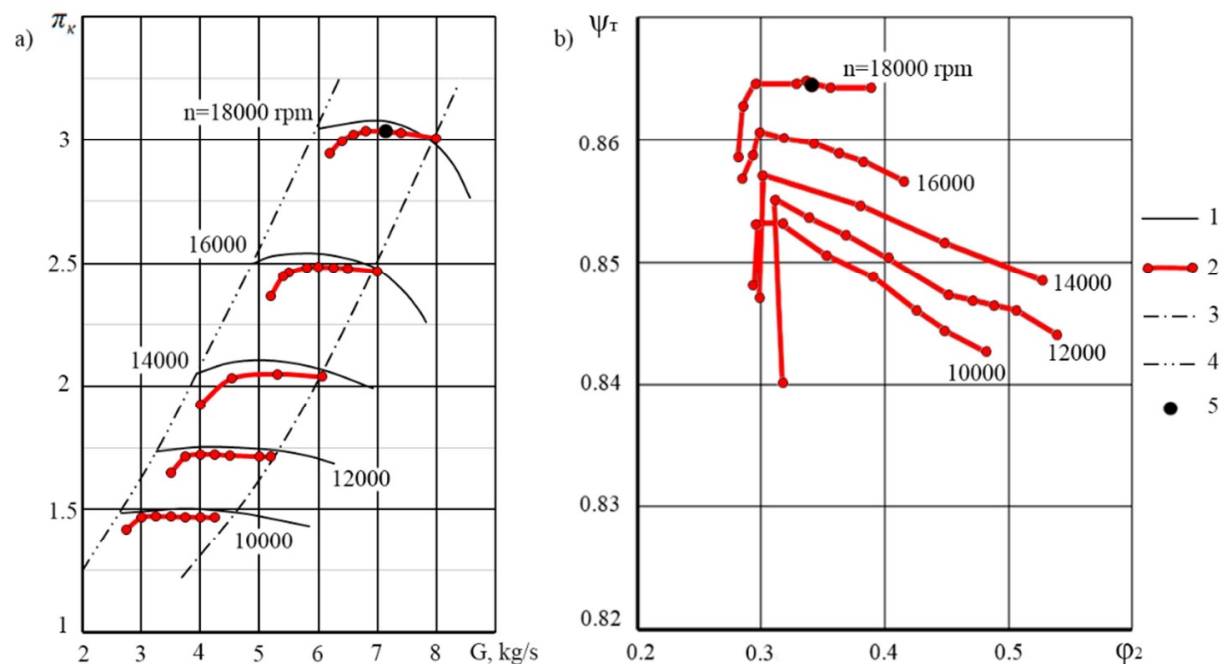

Fig. 2. a) Compressor overall performance in terms of mass flow versus pressure ratio b) Performance characteristic in terms of loading coefficient versus flow coefficient. 1- experimental speed lines, 2 computed speed lines, 3 - throttle curve, 4 - surge limit, 5 - computed design point $\mathrm{G}=7.16 \mathrm{~kg} / \mathrm{s}$, $\mathrm{n}=18000 \mathrm{rpm}$.

Figure $2 \mathrm{~b}$ shows compressor loading coefficient as a function of a flow coefficient $\psi_{T}=f\left(\varphi_{2}\right)$. The dependences are linear and loading coefficient changes slightly. This indicates that compressor total head changes insignificantly with varying mass flow. Considering this together with figure 1a) gives us that increasing Euler work is being compensated by increasing losses, and when the pressure ratio goes down near surge, the pressure losses become significantly bigger, especially at the diffuser (based on flow analysis). This effect in the computation is caused by flow behavior prediction especially next to the walls. When the flow separation is predicted, sharp increase of the deviation angle $\varepsilon$ occur, followed by reduction of circumferential absolute velocity $C_{u}$, and therefore reduction of Euler's work. Similar findings were presented in study [5].

Figure 3 shows the comparison of velocity diagrams at the design operating point. Parameters are considered both for computational and experimental results at the shroud section. The averaging of the kinematic parameters is performed by mass flow.

In the numerical simulations, the axial velocity is greater than in the experiment. This fact leads to a low incidence angle and deviation angle. The values of the circumferential absolute velocity at the outlet impeller $C_{2 u}$ in the numerical simulation are greater than in the experiment by $4.6 \%$, which increases Euler's work as compared with the experiment.
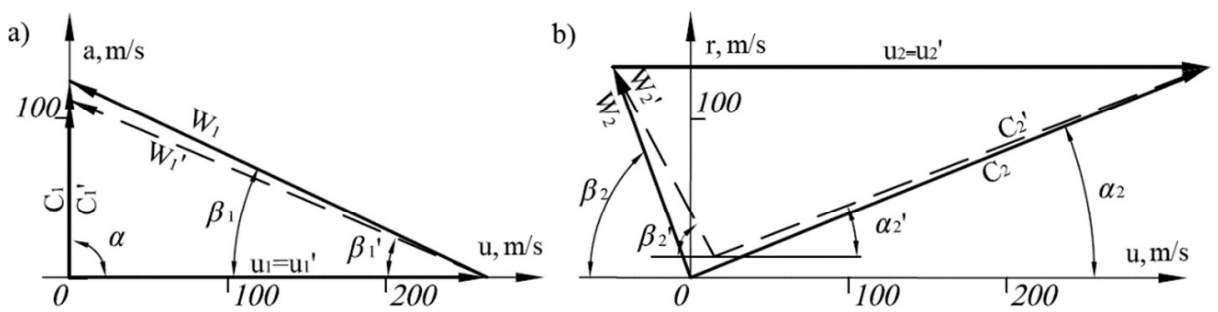

Fig. 3. Velocity diagram in the Eckardt experiment (dotted line) and in the numerical simulation (solid line). a) inlet velocity triangle b) outlet velocity triangle.

Compressor pressure ratio in numerical simulation $\pi_{\kappa, 04 p}=3.035$ is less than the experimental value $\pi_{\kappa, 043}=3.070$ by $1.15 \%$. 


\section{Conclusion}

The results of the comparison between computational results and experimental data show the need for a more detailed study of the internal flow structure to explain the following fundamental deviations.

1. Computational model failed to predict the level of stall margin: the last converging point lays far right from the experimental border. Computed stall margin at the design shaft speed shown to be less by $43 \%$.

2. The computed compressor speed lines shown lower pressure ratio. The convergence between computed and experimental speed lines is significantly better at low shaft speeds. With greater rpm a slope of the speed lines starts to differ from experimental ones, so the change in the pressure ratio gets bigger when approaching stall.

3. The compressor efficiency is overly predicted in the computational model apart from those near stall. The higher the shaft speed the bigger deviation was observed. This could be also caused by incorrect prediction of the shock losses at high rotational speeds since the Mach number at such conditions reaches the value of 1.11 .

4. Additionally, the comparison between the design velocity diagram at the inlet shroud with computational results was shown. Even though the computed values of total mass flow and pressure ratio are identical to those in the experiment, there is a noticeable difference between the diagrams, which might be explained by non-uniform radial flow distribution at the impeller inlet in the model.

All this requires a more detailed study of local flow features and overall flow structure in the flow path.

\section{Nomenclature}

$\pi_{k}$-pressure ratio, $G$-mass flow rate, n-rotational velocity, $\varphi_{2}$-flow coefficient, $\delta=\beta_{\text {geo }}-\beta$ deviation angle, $\psi_{T}$-loading coefficient, $W$-relative velocity, $C$-absolute velocity, $\alpha$-absolute direction, $\beta$-relative direction, $a$-axial axis, $r$-radial axis, $u$-circumferential axis.

Subscripts: 1 -impeller inlet, 2 -impeller exit, '-experimental value.

\section{References}

1. N.A. Cumpsty, Compressor aerodynamic (2000)

2. O.V. Komarov, V.A. Sedunin, V.L. Blinov, S.A. Serkov, International Journal of Gas Turbine, Optimization approach and some results for $2 d$ compressor airfoil, Propulsion and Power Systems, 3 (2016).

3. Y.B. Galerkin, V.P. Mitrofanov, A.Y. Prokofiev, Proceedings of the International Gas Turbine Congress, The experience of CFD calculations for flow analysis in centrifugal compressor stages, (Tokyo, 2003)

4. D. Eckardt, Investigation of the jet-wake flow of a highly-loaded centrifugal compressor impeller Doctoral Dissertation, (Aachen, 1977)

5. Y.B. Galerkin, A.N. Karpov, Kompressornaya tekhnika i pnevmatika, Development of method for centrifugal compressor impeller pressure characteristic modeling on results of model stages tests, $\mathbf{6}$ (2011) 